\title{
Some Properties of Serre Subcategories in the Graded Local Cohomology Modules
}

\author{
Feysal Hassani and Rasul Rasuli \\ Department of Mathematics, Payame Noor University (PNU), Tehran, Iran \\ Correspondence should be addressed to Feysal Hassani; feysal.hassani.pnu@gmail.com
}

Received 11 February 2017; Accepted 7 May 2017; Published 3 July 2017

Academic Editor: Ali Jaballah

Copyright (C) 2017 Feysal Hassani and Rasul Rasuli. This is an open access article distributed under the Creative Commons Attribution License, which permits unrestricted use, distribution, and reproduction in any medium, provided the original work is properly cited.

\begin{abstract}
Let $R=\oplus_{n \geq 0} R_{n}$ be a standard homogeneous Noetherian ring with local base ring $\left(R_{0}, \mathfrak{m}_{0}\right)$ and let $M$ be a finitely generated graded $R$-module. Let $H_{R_{+}}^{i}(M)$ be the $i$ th local cohomology module of $M$ with respect to $R_{+}=\oplus_{n>0} R_{n}$. Let $\mathcal{S}$ be a Serre subcategory of the category of $R$-modules and let $i$ be a nonnegative integer. In this paper, if $\operatorname{dim} R_{0} \leq 1$, then we investigate some conditions under which the $R$-modules $R_{0} / \mathfrak{m}_{0} \otimes_{R_{0}} H_{R_{+}}^{i}(M), \Gamma_{\mathfrak{m}_{0} R}\left(H_{R_{+}}^{i}(M)\right)$ and $H_{\mathfrak{m}_{0} R}^{1}\left(H_{R_{+}}^{i}(M)\right)$ are in $\mathcal{S}$ for all $i \geq 0$. Also, we prove that if $\operatorname{dim} R_{0} \leq 2$, then the graded $R$-module $H_{\mathfrak{m}_{0}}^{1}\left(H_{R_{+}}^{i}(M)\right)$ is in $\mathcal{S}$ for all $i \geq 0$. Finally, we prove that if $n$ is the biggest integer such that $H_{\mathfrak{a}}^{i}(M) \notin \mathcal{S}$, then $H_{R_{+}}^{i}(M) / \mathfrak{m}_{0} H_{R_{+}}^{i}(M) \in \mathcal{S}$ for all $i \geq n$.
\end{abstract}

\section{Introduction}

Let $R=\oplus_{n \geq 0} R_{n}$ be a Noetherian homogeneous ring with local base ring $\left(R_{0}, \mathfrak{m}_{0}\right)$. So, $R_{0}$ is a Noetherian ring and there are finitely many elements $l_{1}, l_{2}, \ldots, l_{r} \in R_{1}$ such that $R=$ $R_{0}\left[l_{1}, l_{2}, \ldots, l_{r}\right]$. Let $R_{+}=\oplus_{n>0} R_{n}$ denote the irrelevant ideal of $R$ and let $\mathfrak{m}=\mathfrak{m}_{0} \oplus R_{+}$denote the graded maximal ideal of $R$. Finally, let $M=\oplus_{n \in \mathbb{Z}} M_{n}$ be a finitely generated graded $R$-module. Recall that a class of $R$-modules is a Serre subcategory of the category of $R$-modules when it is closed under taking submodules, quotients, and extensions. Always, $\mathcal{S}$ stands for a Serre subcategory of the category of $R$-modules. Hence, if $L \rightarrow M \rightarrow N$ is an exact sequence of the category of $R$-modules and $R$-homomorphisms such that both end terms belong to $\mathcal{S}$, then $M$ also belongs to $\mathcal{\delta}$. Note that the following subcategories are examples of Serre subcategory of the category of $R$-modules: finite $R$-modules; coatomic $R$ modules [1]; minimax $R$-modules [2]; $\mathfrak{a}$-cofinite $R$-modules; weakly Laskerian $R$-modules; Matlis reflexive $R$-modules; and trivially the zero $R$-modules. Recently, some results have been proved concerning the local cohomology modules $H_{\mathfrak{a}}^{i}(M)$ of a module $M$ in some certain Serre subcategory of the category of modules (cf. [3-7]). Aghapournahr and Melkersson (cf. [4]) gave a condition on a Serre subcategory
$\mathcal{S}$. To give more details, let $\mathfrak{a}$ be an ideal of $R$, let $M$ be an $R$ module, and let $\left(0::_{M} \mathfrak{a}\right)=\{x \in M \mid \mathfrak{a} x=0\}$. The $R$-module $M$ is said to satisfy $C_{a}$ condition on $\mathcal{S}$ whenever the following condition holds:

If $M$

$=\Gamma_{\mathfrak{a}}(M)$ and if $\left(0:_{M} \mathfrak{a}\right)$ is in $\mathcal{S}$ then $M$ is in $\mathcal{S}$.

Then $\mathcal{S}$ is said to satisfy $C_{\mathrm{a}}$ condition, whenever, every $R$ module satisfies $C_{\mathfrak{a}}$ condition on $\mathcal{S}$. For example, the class of zero modules and Artinian $R$-modules satisfy the condition $C_{\mathfrak{a}}$.

In this paper, according to $\operatorname{dim} R_{0} \leq 1$, we investigate some conditions under which the $R$-modules $R_{0} / \mathfrak{m}_{0} \otimes_{R_{0}} H_{R_{+}}^{i}(M), \Gamma_{\mathfrak{m}_{0} R}\left(H_{R_{+}}^{i}(M)\right)$ and $H_{\mathfrak{m}_{0} R}^{1}\left(H_{R_{+}}^{i}(M)\right)$ are in $\mathcal{S}$ for all $i \geq 0$. Next, we prove that if $\operatorname{dim} R_{0} \leq 2$, then $H_{\mathfrak{m}_{0}}^{1}\left(H_{R_{+}}^{i}(M)\right)$ is in $\mathcal{S}$ for all $i \geq 0$. Also if $\mathcal{S}$ satisfies $C_{a}$ condition, then under some conditions, $R$-modules $H_{R_{+}}^{i}\left(H_{\mathfrak{m}_{0} R}^{1}(M)\right)$ and $\Gamma_{R_{+}}\left(H_{\mathfrak{m}_{0} R}^{1}(M)\right)$ belong to $\mathcal{S}$. Finally, we prove that if $n$ is the biggest integer such that $H_{\mathfrak{a}}^{i}(M) \notin \mathcal{S}$, then $H_{R_{+}}^{i}(M) / \mathfrak{m}_{0} H_{R_{+}}^{i}(M) \in \mathcal{S}$ for all $i \geq n$.

In Section 2, we give some notations and definition that we used in Section 3. 


\section{Preliminaries}

Let $R$ be a ring. The spectrum of $R$ is denoted by $\operatorname{Spec} R$, that is, the set of prime ideals of $R$ with the Zariski topology, which is the topology where the closed sets are $V(\mathfrak{a})=\{\mathfrak{p} \in \operatorname{Spec} R \mid$ $\mathfrak{a} \subseteq \mathfrak{p}\}$ for ideals $\mathfrak{a} \subseteq \mathfrak{p}$.

The height of a prime ideal $\mathfrak{p}$, denoted by height $\mathfrak{p}$, is defined by the supremum of integers $t$ such that there exists a chain of prime ideals $\mathfrak{p}=\mathfrak{p}_{0} \supsetneq \mathfrak{p}_{1} \supsetneq \mathfrak{p}_{2} \supsetneq \cdots \supsetneq \mathfrak{p}_{\mathfrak{t}}$, where $\mathfrak{p}_{\mathfrak{i}} \in \operatorname{Spec} R$. The Krull dimension of $R$ is defined as $\operatorname{dim} R=\sup \{$ height $\mathfrak{p} \mid \mathfrak{p} \in \operatorname{Spec} R\}$.

Consider the complex $K^{\bullet}$

$$
\cdots \longrightarrow K^{i-1} \stackrel{\delta_{K}^{i-1}}{\longrightarrow} K^{i} \stackrel{\delta_{K}^{i}}{\longrightarrow} K^{i+1} \longrightarrow \cdots .
$$

The differential on $K^{\bullet}$ is denoted by $\delta_{K}$, or sometimes simply $\delta$. We say that $K^{\bullet}$ is bounded below if $K^{i}=0$ for $i \ll 0$ and bounded above if $K^{i}=0$ for $i \gg 0$; if both conditions hold, then $K^{\bullet}$ is bounded. On occasion, we consider a complex as a graded module with a graded endomorphism of degree 1.

Let $M$ be an $R$-module. An injective resolution of $M$ is a complex $E^{\bullet}$ of injective $R$-modules $0 \rightarrow E^{0} \rightarrow E^{1} \rightarrow$ $E^{2} \rightarrow \cdots$, equipped with a quasi-isomorphism of complexes $l: M \rightarrow E^{\bullet}$; here one views $M$ as a complex concentrated in degree 0 . Said otherwise, a complex $E^{\bullet}$ of injective modules is a resolution of $M$ if there is an exact sequence $0 \rightarrow M \stackrel{l}{\rightarrow}$ $E^{0} \rightarrow E^{1} \rightarrow E^{2} \rightarrow \cdots$.

Let $M$ and $N$ be $R$-modules. Then $\left\{\operatorname{Ext}_{R}^{i}(M,-)\right\}_{i \geq 0}$ are the right derived functors of $\operatorname{Hom}_{R}(M,-)$, and $\left\{\operatorname{Tor}_{i}^{R}(-, N)\right\}_{i \geq 0}$ are the left derived functors of $-\otimes_{R} N$.

For an exact sequence of $R$-modules $0 \rightarrow J \rightarrow M \rightarrow L \rightarrow$ 0 and each $R$-module $N$, one has exact sequences:

$$
\begin{aligned}
\cdots & \longrightarrow \operatorname{Ext}_{R}^{i}(N, M) \longrightarrow \operatorname{Ext}_{R}^{i}(N, L) \longrightarrow \operatorname{Ext}_{R}^{i+1}(N, J) \\
& \longrightarrow \operatorname{Ext}_{R}^{i+1}(N, M) \longrightarrow \cdots, \\
\cdots & \longrightarrow \operatorname{Ext}_{R}^{i}(M, N) \longrightarrow \operatorname{Ext}_{R}^{i}(J, N) \longrightarrow \operatorname{Ext}_{R}^{i+1}(L, N) \\
& \longrightarrow \operatorname{Ext}_{R}^{i+1}(M, N) \longrightarrow \cdots, \\
\cdots & \longrightarrow \operatorname{Tor}_{i+1}^{R}(M, N) \longrightarrow \operatorname{Tor}_{i+1}^{R}(L, N) \\
& \longrightarrow \operatorname{Tor}_{i}^{R}(J, N) \longrightarrow \operatorname{Tor}_{i}^{R}(M, N) \longrightarrow \cdots .
\end{aligned}
$$

For an $R$-module $M$, the $\mathfrak{a}$-torsion of $M$ is defined by $\Gamma_{\mathfrak{a}}(M):=\bigcup_{n \in \mathbb{N}}\left(0:_{M} \mathfrak{a}^{\mathfrak{n}}\right)$. Observe that $\Gamma_{\mathfrak{a}}(M)$ is a submodule of $M$ and $\Gamma_{\mathfrak{a}}(-)$ is a left exact $\mathfrak{a}$-torsion functor of $R$-modules and $R$-homomorphisms. Let $n \in \mathbb{N}_{0}$ and let $M$ be an $R$ module. Choose an injective resolution of $M$, so that we have an exact sequence

$$
0 \longrightarrow M \stackrel{l}{\longrightarrow} E^{0} \stackrel{d^{0}}{\longrightarrow} E^{1} \stackrel{d^{1}}{\longrightarrow} E^{2} \stackrel{d^{2}}{\longrightarrow} E^{3} \longrightarrow \cdots
$$

Then, apply the functor $\Gamma_{\mathfrak{a}}(-)$ to the resolving cocomplex

$$
\cdots \longrightarrow 0 \stackrel{d^{-1}}{\longrightarrow} E^{0} \stackrel{d^{0}}{\longrightarrow} E^{1} \stackrel{d^{1}}{\longrightarrow} E^{2} \stackrel{d^{2}}{\longrightarrow} E^{3} \longrightarrow \cdots
$$

in order to obtain the cocomplex

$$
\begin{aligned}
\cdots & \longrightarrow 0 \stackrel{\Gamma_{\mathfrak{a}}\left(d^{-1}\right)}{\longrightarrow} \Gamma_{\mathfrak{a}}\left(E^{0}\right) \stackrel{\Gamma_{\mathfrak{a}}\left(d^{0}\right)}{\longrightarrow} \Gamma_{\mathfrak{a}}\left(E^{1}\right) \stackrel{\Gamma_{\mathfrak{a}}\left(d^{1}\right)}{\longrightarrow} \Gamma_{\mathfrak{a}}\left(E^{2}\right) \\
& \stackrel{\Gamma_{\mathfrak{a}}\left(d^{2}\right)}{\longrightarrow} \cdots .
\end{aligned}
$$

Then, the $n$th local cohomology module of $M$ with respect to $\mathfrak{a}$ is defined as the $R$-module $H_{\mathfrak{a}}^{n}(M)=$ $\operatorname{ker}\left(\Gamma_{\mathfrak{a}}\left(d^{n}\right)\right) / \operatorname{Im}\left(\Gamma_{\mathfrak{a}}\left(d^{n-1}\right)\right)$. If $0 \rightarrow L \stackrel{f}{\rightarrow} M \stackrel{g}{\rightarrow} N \rightarrow 0$ is an exact sequence of $R$-modules and $R$-homomorphisms, then we have the following long exact sequence:

$$
\begin{aligned}
0 \longrightarrow H_{\mathfrak{a}}^{0}(L) \stackrel{H_{\mathfrak{a}}^{0}(f)}{\longrightarrow} H_{\mathfrak{a}}^{0}(M) \stackrel{H_{\mathfrak{a}}^{0}(g)}{\longrightarrow} H_{\mathfrak{a}}^{0}(N) \longrightarrow H_{\mathfrak{a}}^{1}(L) \\
\stackrel{H_{\mathfrak{a}}^{1}(f)}{\longrightarrow} H_{\mathfrak{a}}^{1}(M) \stackrel{H_{\mathfrak{a}}^{1}(g)}{\longrightarrow} H_{\mathfrak{a}}^{1}(N) \longrightarrow \cdots .
\end{aligned}
$$

As a general reference to homological and commutative algebra, we refer to [8].

\section{Main Results}

In this paper, $R=\bigoplus_{n \geq 0} R_{n}$ is a Noetherian homogeneous ring with local base ring $\left(R_{0}, \mathfrak{m}_{0}\right)$. We start off this section with some technical lemmas to rich our main aims in this work.

Lemma 1. Let $H \in \mathcal{S}$ be an $R$-module and let $M_{0}$ be a finitely generated $R_{0}$-module. Then for any $i \geq 0$ the $R$-module $\operatorname{Tor}_{i}^{R_{0}}\left(M_{0}, H\right) \in \mathcal{S}$.

Proof. Let

$$
\begin{aligned}
\cdots & \longrightarrow F_{i+1} \longrightarrow F_{i} \longrightarrow F_{i-1} \longrightarrow \cdots \longrightarrow F_{1} \longrightarrow F_{0} \\
& \longrightarrow M \longrightarrow 0
\end{aligned}
$$

be a minimal free resolution of $M_{0}$. If $F_{i}=R^{n_{i}}$ for some integer $n_{i}$, then $\operatorname{Tor}_{i}^{R_{0}}\left(M_{0}, H\right)$ is an $R_{0}$-subquotient of $F_{i} \otimes_{R_{0}} H \cong H^{n_{i}}$. Thus $\operatorname{Tor}_{i}^{R_{0}}\left(M_{0}, H\right) \in \mathcal{S}$.

Lemma 2. If $M, R / \mathfrak{a} \in \mathcal{S}$, then $H_{\mathfrak{a}}^{i}(M) \in \mathcal{S}$ for all $i \geq 0$.

Proof. Let $F_{0}: \cdots \rightarrow F_{1} \rightarrow F_{0} \rightarrow 0$ be a finite free resolution of $R / \mathfrak{a}^{n}$. If $F_{i}=R^{n_{i}}$ for some integer $n_{i}$, then $\operatorname{Ext}_{R}^{i}\left(R / \mathfrak{a}^{n}, M\right)=$ $H^{i}\left(\operatorname{Hom}_{R}\left(F_{\bullet}, M\right)\right)$ is a subquotient of $M^{n_{i}}$. Since $\delta$ is a Serre class, $\operatorname{Ext}_{R}^{i}\left(R / \mathfrak{a}^{n}, M\right) \in \mathcal{S}$. From definition, we have $H_{\mathfrak{a}}^{i}(M)=$ $\lim _{n \in N} \operatorname{Ext}_{R}^{i}\left(R / \mathfrak{a}^{n}, M\right)$ and so $H_{\mathfrak{a}}^{i}(M) \in \mathcal{S}$ for all $i \geq 0$.

An immediate result of the above lemmas is the following result.

Corollary 3. If $\operatorname{dim}\left(R_{0}\right)=0$ and $M \in \mathcal{S}$, then $R_{0}=R / R_{+}$is in $\mathcal{S}$ and $H_{R_{+}}^{i}(M) \in \mathcal{S}$ for all $i \geq 0$.

Lemma 4. If $M \in \mathcal{S}$, then $H_{R_{+}}^{i}\left(\Gamma_{\mathfrak{m}_{0} R}(M)\right) \in \mathcal{S}$ as an $R$ module for all $i \geq 0$. 
Proof. Since $\Gamma_{\mathfrak{m}_{0} R}(M)$ is a finitely generated $\mathfrak{m}_{0}$-torsion module, there exists an $n \geq 0$ such that $\mathfrak{m}_{0}^{n} \Gamma_{\mathfrak{m}_{0} R}(M)=0$. Then we obtain the $R / \mathfrak{m}_{0}^{n}$-module $\Gamma_{\mathfrak{m}_{0} R}(M)$. If $i \geq 0$, then from the Graded Independence Theorem (cf. ([8], 13.1.6)) there is an isomorphism of graded $R$-modules $H_{R_{+}}^{i}\left(\Gamma_{\mathfrak{m}_{0} R}(M)\right) \cong$ $H_{\left(R / \mathfrak{m}_{0}^{n} R\right)_{+}}^{i}\left(\Gamma_{\mathfrak{m}_{0} R}(M)\right)$. Since $\operatorname{dim}\left(R / \mathfrak{m}_{0}^{n} R\right)_{0}=\operatorname{dim} R_{0} / \mathfrak{m}_{0}^{n}=0$, Corollary 3 implies that $H_{R_{+}}^{i}\left(\Gamma_{\mathfrak{m}_{0} R}(M)\right) \in \mathcal{S}$.

Lemma 5. Let $i \geq 0$ and $R_{0} / \mathfrak{m}_{0} \otimes_{R_{0}} H_{R_{+}}^{i}\left(M / \Gamma_{\mathfrak{m}_{0} R}(M)\right) \in \mathcal{S}$; then $R_{0} / \mathfrak{m}_{0} \otimes_{R_{0}} H_{R_{+}}^{i}(M) \in \mathcal{S}$.

Proof. Let $L:=M / \Gamma_{\mathfrak{m}_{0} R}(M)$. Then we have the exact rows:

$$
\begin{aligned}
H_{R_{+}}^{i}\left(\Gamma_{\mathfrak{m}_{0} R}(M)\right) & \stackrel{f}{\longrightarrow} H_{R_{+}}^{i}(M) \stackrel{g}{\longrightarrow} H_{R_{+}}^{i}(L) \\
& \longrightarrow H_{R_{+}}^{i+1}\left(\Gamma_{\mathfrak{m}_{0} R}(M)\right), \\
0 & \longrightarrow \frac{H_{R_{+}}^{i}(M)}{\operatorname{Im} f} \longrightarrow H_{R_{+}}^{i}(L) \\
& \longrightarrow \frac{H_{R_{+}}^{i}(L)}{\operatorname{Im} g} \longrightarrow 0,
\end{aligned}
$$

such that $h: H_{R_{+}}^{i}(M) \rightarrow H_{R_{+}}^{i}(M) / \operatorname{Im} f$ is an epimorphism and $k: H_{R_{+}}^{i}(L) / \operatorname{Im} g \rightarrow H_{R_{+}}^{i+1}\left(\Gamma_{\mathrm{m}_{0} R}(M)\right)$ is a monomorphism. Since $T:=H_{R_{+}}^{i}(L) / \operatorname{Im} g$ is a submodule of $H_{R_{+}}^{i+1}\left(\Gamma_{\mathfrak{m}_{0} R}(M)\right)$, Lemma 4 leads to $T \in \mathcal{S}$. Also, from Lemma 1, we get $\operatorname{Tor}_{1}^{R_{0}}\left(R_{0} / \mathfrak{m}_{0}, T\right) \in \mathcal{S}$. The short exact sequence (10) deduces the following exact sequence:

$$
\begin{aligned}
\operatorname{Tor}_{1}^{R_{0}}\left(\frac{R_{0}}{\mathfrak{m}_{0}}, T\right) & \longrightarrow \frac{R_{0}}{\mathfrak{m}_{0}} \otimes_{R_{0}} \frac{H_{R_{+}}^{i}(M)}{\operatorname{Im} f} \\
& \longrightarrow \frac{R_{0}}{\mathfrak{m}_{0}} \otimes_{R_{0}} H_{R_{+}}^{i}(L) .
\end{aligned}
$$

According to our assumption, $R_{0} / \mathfrak{m}_{0} \otimes_{R_{0}} H_{R_{+}}^{i}(L) \in \mathcal{S}$ and then $R_{0} / \mathfrak{m}_{0} \otimes_{R_{0}} H_{R_{+}}^{i}(M) / \operatorname{Im} f \in \mathcal{S}$. From the exact sequence (9), we have the exact sequence $R_{0} / \mathfrak{m}_{0} \otimes_{R_{0}} H_{R_{+}}^{i}(M /$ $\left.\Gamma_{\mathfrak{m}_{0} R}(M)\right) \rightarrow R_{0} / \mathfrak{m}_{0} \otimes_{R_{0}} H_{R_{+}}^{i}(M) \rightarrow R_{0} / \mathfrak{m}_{0} \otimes_{R_{0}} H_{R_{+}}^{i}(M) /$ $\operatorname{Im} f$, and hence $R_{0} / \mathfrak{m}_{0} \otimes_{R_{0}} H_{R_{+}}^{i}(M) \in \mathcal{S}$.

Theorem 6. Let $\operatorname{dim} R_{0} \leq 1$ and $i \geq 0$; then the following assertions hold:

(1) If $M \in \mathcal{S}$, then $R_{0} / \mathfrak{m}_{0} \otimes_{R_{0}} H_{R_{+}}^{i}(M) \in \mathcal{S}$.

(2) If $x \in \mathfrak{m}_{0}$, such that $\sqrt{x R_{0}}=\mathfrak{m}_{0}$, and $H_{\left(R_{+}, x\right)}^{i}(M) \in \mathcal{S}$, then the R-modules $\Gamma_{\mathfrak{m}_{0} R}\left(H_{R_{+}}^{i}(M)\right)$ and $H_{\mathrm{m}_{0} R}^{1}\left(H_{R_{+}}^{i}(M)\right)$ are in $\mathcal{S}$.

Proof. (1) If $\operatorname{dim} R_{0}=0$, then from Corollary 3 we have $H_{R_{+}}^{i}(M) \in \mathcal{S}$ and then from Lemma 1 we get $R_{0} / \mathfrak{m}_{0} \otimes_{R_{0}} H_{R_{+}}^{i}(M) \in \mathcal{S}$. Let $\operatorname{dim} R_{0}=1$ and $\Gamma_{\mathfrak{m}_{0} R}(M)=0$.
Then there exists an $M$-regular element $x \in \mathfrak{m}_{0}$ which avoids all minimal primes of $R_{0}$. Now, the exact sequence

$$
0 \longrightarrow M \stackrel{x}{\longrightarrow} M \longrightarrow \frac{M}{x M} \longrightarrow 0
$$

induces an exact sequence

$$
\begin{gathered}
H_{R_{+}}^{i-1}\left(\frac{M}{x M}\right) \longrightarrow H_{R_{+}}^{i}(M) \stackrel{x}{\longrightarrow} H_{R_{+}}^{i}(M) \\
\stackrel{f}{\longrightarrow} H_{R_{+}}^{i}\left(\frac{M}{x M}\right) .
\end{gathered}
$$

Since $x$ avoids all minimal primes of $R_{0}, \operatorname{dim} R_{0} / x R_{0}=$ 0 . Then by the Independence Theorem, $H_{R_{+}}^{i}(M / x M) \in \mathcal{S}$ and then $\operatorname{Im} f \in \mathcal{S}$. The exact sequence (13) gives the exact sequence

$$
\begin{aligned}
\frac{R_{0}}{\mathfrak{m}_{0}} \otimes_{R_{0}} H_{R_{+}}^{i}(M) & \stackrel{x}{\longrightarrow} \frac{R_{0}}{\mathfrak{m}_{0}} \otimes_{R_{0}} H_{R_{+}}^{i}(M) \\
& \longrightarrow \frac{R_{0}}{\mathfrak{m}_{0}} \otimes_{R_{0}} \operatorname{Im} f \longrightarrow 0 .
\end{aligned}
$$

As $x \in \mathfrak{m}_{0}$, the multiplication map $x$ is zero. Then there is an isomorphism

$$
\frac{R_{0}}{\mathfrak{m}_{0}} \otimes_{R_{0}} H_{R_{+}}^{i}(M) \cong \frac{R_{0}}{\mathfrak{m}_{0}} \otimes_{R_{0}} \operatorname{Im} f .
$$

Therefore, $R_{0} / \mathbf{m}_{0} \otimes_{R_{0}} H_{R_{+}}^{i}(M) \in \mathcal{S}$.

(2) Let $x \in \mathfrak{m}_{0}$ such that $\sqrt{x R_{0}}=\mathfrak{m}_{0}$. Therefore, for all $i \geq 0$, there is an exact sequence of graded $R$-modules

$$
\begin{gathered}
H_{R_{+}}^{i-1}(M) \stackrel{f_{x}^{i-1}}{\longrightarrow} H_{R_{+}}^{i-1}(M)_{x} \longrightarrow H_{\left(R_{+}, x\right)}^{i}(M) \\
\longrightarrow H_{R_{+}}^{i}(M) \stackrel{f_{x}^{i}}{\longrightarrow} H_{R_{+}}^{i}(M)_{x}
\end{gathered}
$$

such that $f_{x}^{i-1}$ and $f_{x}^{i}$ are the natural homomorphisms (cf. [8], 13.1.12). Now from

$$
\begin{aligned}
& \operatorname{coker}\left(f_{x}^{i-1}\right) \cong H_{x R}^{1}\left(H_{R_{+}}^{i}(M)\right) \cong H_{\mathfrak{m}_{0} R}^{1}\left(H_{R_{+}}^{i}(M)\right), \\
& \operatorname{ker}\left(f_{x}^{i}\right) \cong \Gamma_{x R}\left(H_{R_{+}}^{i}(M)\right)=\Gamma_{\mathfrak{m}_{0} R}\left(H_{R_{+}}^{i}(M)\right),
\end{aligned}
$$

we obtain an exact sequence

$$
\begin{aligned}
0 & \longrightarrow H_{\mathfrak{m}_{0} R}^{1}\left(H_{R_{+}}^{i-1}(M)\right) \longrightarrow H_{\left(R_{+}, x\right)}^{i}(M) \\
& \longrightarrow \Gamma_{\mathfrak{m}_{0} R}\left(H_{R_{+}}^{i}(M)\right) \longrightarrow 0
\end{aligned}
$$

Therefore, $\Gamma_{\mathfrak{m}_{0} R}\left(H_{R_{+}}^{i}(M)\right)$ and $H_{\mathfrak{m}_{0} R}^{1}\left(H_{R_{+}}^{i}(M)\right)$ are in $\mathcal{S}$.

Corollary 7. Let $\operatorname{dim} R_{0} \leq 1$ and $i \geq 0$. Moreover, let $\mathfrak{q}_{0} \subseteq R_{0}$ be an $\mathfrak{m}_{0}$-primary ideal. Then

(1) the R-module $R_{0} / \mathfrak{m}_{0} / \mathfrak{q}_{0} H_{R_{+}}^{i}(M)$ is in $\mathcal{S}$;

(2) the R-module $\left(0:_{H_{R_{+}}^{i}(M)} \mathfrak{q}_{0}\right)$ is in $\mathcal{S}$. 
Proof. (1) Since $\mathfrak{q}_{0}$ is an $\mathfrak{m}_{0}$-primary ideal, there is some $n \geq 0$ such that $\mathfrak{m}_{0}^{n} \subseteq \mathfrak{q}_{0}$. Then it suffices to prove that $R_{0} / \mathfrak{m}_{0}^{n} \otimes_{R_{0}} H_{R_{+}}^{i}(M) \in \mathcal{S}$. We proceed the assertion by induction on $n$. For $n=1$, use part (1) of Theorem 6 . Now suppose, inductively, that $n>1$ and the result has been proved for all values smaller than $n$. We have the exact sequence

$$
\begin{aligned}
\frac{\mathfrak{m}_{0}^{n-1}}{\mathfrak{m}_{0}^{n}} \otimes_{R_{0}} H_{R_{+}}^{i}(M) & \longrightarrow \frac{R_{0}}{\mathfrak{m}_{0}^{n}} \otimes_{R_{0}} H_{R_{+}}^{i}(M) \\
& \longrightarrow \frac{R_{0}}{\mathfrak{m}_{0}^{n-1}} \otimes_{R_{0}} H_{R_{+}}^{i}(M),
\end{aligned}
$$

and the natural isomorphism

$$
\begin{aligned}
\frac{\mathfrak{m}_{0}^{n-1}}{\mathfrak{m}_{0}^{n}} \otimes_{R_{0}} H_{R_{+}}^{i}(M) \cong & \mathfrak{m}_{0}^{n-1} \\
& \otimes_{R_{0}}\left(\frac{R_{0}}{\mathfrak{m}_{0}} \otimes_{R_{0}} H_{R_{+}}^{i}(M)\right) .
\end{aligned}
$$

Now, use part (1) of Theorem 6 and Lemma 1.

(2) Since $\left(0:_{H_{R_{+}}^{i}(M)} \mathfrak{q}_{0}\right) \subseteq \Gamma_{\mathfrak{m}_{0} R}\left(H_{R_{+}}^{i}(M)\right)$, part (2) of Theorem 6 implies that $\left(0:_{H_{R_{+}}^{i}(M)} \mathfrak{q}_{0}\right) \in \mathcal{S}$.

The following result is an extension of Theorem 6 to the case $\operatorname{dim} R_{0}=2$.

Theorem 8. If $\operatorname{dim} R_{0} \leq 2$, then $H_{\mathfrak{m}_{0}}^{1}\left(H_{R_{+}}^{i}(M)\right) \in \mathcal{S}$ for all $i \geq 0$.

Proof. If $\operatorname{dim} R_{0} \leq 1$, this statement is the same as Theorem 6 . Let $\operatorname{dim} R_{0}=2$, then there is a system of parameters $(x, y)$ of $R_{0}$ such that $H_{\mathfrak{m}_{0} R}^{1}\left(H_{R_{+}}^{i}(M)\right)=H_{(x, y) R}^{1}\left(H_{R_{+}}^{i}(M)\right)$. From the exact sequence

$$
\begin{aligned}
0 & \longrightarrow H_{y R}^{1}\left(H_{R_{+}}^{i-1}(M)\right) \longrightarrow H_{\left(y, R_{+}\right)}^{i}(M) \\
& \longrightarrow \Gamma_{y R}\left(H_{R_{+}}^{i}(M)\right) \longrightarrow 0,
\end{aligned}
$$

which obtains an epimorphism $H_{x R}^{1}\left(H_{\left(y, R_{+}\right)}^{i}(M)\right) \rightarrow$ $H_{x R}^{1}\left(\Gamma_{y R}\left(H_{R_{+}}^{i}(M)\right)\right)$, also, there is a monomorphism

$$
H_{x R}^{1}\left(H_{\left(y, R_{+}\right)}^{i}(M)\right) \longrightarrow H_{\left(x, y, R_{+}\right)}^{i+1}(M)=H_{\mathfrak{m}}^{i+1}(M) .
$$

Since $H_{\mathfrak{m}}^{i+1}(M) \in \mathcal{S}, H_{x R}^{1}\left(\Gamma_{y R}\left(H_{R_{+}}^{i}(M)\right)\right) \in \delta$. Application of the functor $\Gamma_{x R}(-)$ to the monomorphism $H_{y R}^{1}\left(H_{R_{+}}^{i}(M)\right) \rightarrow H_{\left(y, R_{+}\right)}^{i+1}(M)$ yields a monomorphism $\Gamma_{x R}\left(H_{y R}^{1}\left(H_{R_{+}}^{i}(M)\right)\right) \rightarrow \Gamma_{x R}\left(H_{\left(y, R_{+}\right)}^{i+1}(M)\right)$. Also, there is an epimorphism

$$
H_{\mathfrak{m}}^{i+1}(M)=H_{\left(x, y, R_{+}\right)}^{i+1}(M) \longrightarrow \Gamma_{x R}\left(H_{\left(y, R_{+}\right)}^{i+1}(M)\right) .
$$

Because $H_{\mathfrak{m}}^{i+1}(M) \in \mathcal{S}, \Gamma_{x R}\left(H_{y R}^{1}\left(H_{R_{+}}^{i}(M)\right)\right) \in \mathcal{S}$. The exact sequence

$$
\begin{aligned}
0 & \longrightarrow H_{x R}^{1}\left(\Gamma_{y R}\left(H_{R_{+}}^{i}(M)\right)\right) \longrightarrow H_{(x, y) R}^{1}\left(H_{R_{+}}^{i}(M)\right) \\
& \longrightarrow \Gamma_{x R}\left(H_{y R}^{1}\left(H_{R_{+}}^{i}(M)\right)\right) \longrightarrow 0
\end{aligned}
$$

implies that $H_{\mathfrak{m}_{0}}^{1}\left(H_{R_{+}}^{i}(M)\right) \in \mathcal{S}$.
Proposition 9. Let $M \in \mathcal{S}$ and $M$ satisfies $C_{\mathfrak{a}}$ condition on $\mathcal{S}$. If $R_{0}$ is a local ring of dimension one, then $H_{R_{+}}^{i}\left(H_{\mathfrak{m}_{0} R}^{1}(M)\right) \in \mathcal{S}$ for all $i \geq 0$.

Proof. Since $H_{\mathfrak{m}_{0} R}^{1}(M) \cong H_{\mathfrak{m}_{0} R}^{1}\left(M / \Gamma_{\mathfrak{m}_{0} R}(M)\right)$, one can assume that $\Gamma_{\mathfrak{m}_{0} R}(M)=0$. Then, there exists an element $x_{0} \in \mathfrak{m}_{0}$ such that it is an $M$-sequence. By the exact sequence

$$
0 \longrightarrow M \stackrel{x_{0}}{\longrightarrow} M \longrightarrow \frac{M}{x_{0} M} \longrightarrow 0
$$

and by application of the functor $H_{R_{+}}^{i}(-)$ to this exact sequence, we have the following exact sequence

$$
\begin{aligned}
& H_{R_{+}}^{i}\left(\Gamma_{\mathfrak{m}_{0} R}\left(\frac{M}{x_{0} M}\right)\right) \longrightarrow H_{R_{+}}^{i}\left(H_{\mathfrak{m}_{0} R}^{i}(M)\right) \\
& \stackrel{x_{0}}{\longrightarrow} H_{R_{+}}^{i}\left(H_{\mathfrak{m}_{0} R}^{i}(M)\right) \longrightarrow H_{R_{+}}^{i+1}\left(\Gamma_{\mathfrak{m}_{0} R}\left(\frac{M}{x_{0} M}\right)\right) .
\end{aligned}
$$

Lemma 4 leads to $H_{R_{+}}^{i}\left(\Gamma_{\mathfrak{m}_{0} R}\left(M / x_{0} M\right)\right) \in \mathcal{S}$ and then $\left(0:_{H_{R_{+}}^{i}\left(H_{\mathrm{m}_{0} R}^{1}(M)\right)} x_{0}\right) \in \mathcal{S}$. Furthermore, by the basic properties of local cohomology, we have $\Gamma_{\mathfrak{a}}\left(H_{R_{+}}^{i}\left(H_{\mathfrak{m}_{0} R}^{1}(M)\right)\right)=$ $H_{R_{+}}^{i}\left(H_{\mathfrak{m}_{0} R}^{1}(M)\right)$. Since $H_{R_{+}}^{i}\left(H_{\mathfrak{m}_{0} R}^{1}(M)\right)$ satisfies $C_{\mathfrak{a}}$ condition on $\mathcal{S}, H_{R_{+}}^{i}\left(H_{\mathrm{m}_{0} R}^{1}(M)\right) \in \mathcal{S}$ for all $i \geq 0$.

Proposition 10. Suppose that $M$ satisfies $C_{\mathfrak{a}}$ condition on $\mathcal{S}$ and $n$ is the least integer such that $H_{\mathrm{m}_{0} R}^{n}(M) \notin \mathcal{S}$. Then $\Gamma_{R_{+}}\left(H_{\mathfrak{m}_{0} R}^{1}(M)\right) \in \mathcal{S}$ for all $i \leq n$.

Proof. If $i<n$, then $H_{\mathfrak{m}_{0} R}^{i}(M) \in \mathcal{S}$ and therefore $\Gamma_{R_{+}}\left(H_{\mathfrak{m}_{0} R}^{i}(M)\right) \in \mathcal{S}$. Let $i=n$ and from the isomorphism $H_{\mathfrak{m}_{0} R}^{i}(M) \cong H_{\mathfrak{m}_{0} R}^{i}\left(M / \Gamma_{\mathfrak{m}_{0} R}(M)\right)$ for all $i$, one can suppose that $\Gamma_{\mathfrak{m}_{0} R}(M)=0$. Therefore, there exists an element $x \in \mathfrak{m}_{0}$ such that it is an $M$-sequence. In view of the exact sequence

$$
0 \longrightarrow M \stackrel{x}{\longrightarrow} M \longrightarrow \frac{M}{x M} \longrightarrow 0
$$

we have the exact sequence

$$
\begin{aligned}
& H_{\mathfrak{m}_{0} R}^{n-1}(M) \stackrel{f}{\longrightarrow} H_{\mathfrak{m}_{0} R}^{n-1}\left(\frac{M}{x M}\right) \rightarrow H_{\mathfrak{m}_{0} R}^{n}(M) \\
& \stackrel{x}{\longrightarrow} H_{\mathfrak{m}_{0} R}^{n}(M) .
\end{aligned}
$$

By the above sequence, we obtain the exact sequence

$$
0 \longrightarrow \operatorname{ker} f \longrightarrow H_{\mathrm{m}_{0} R}^{n-1}(M) \longrightarrow \operatorname{Im} f \longrightarrow 0,
$$

and isomorphism $\operatorname{Im} f \cong\left(0:_{H_{\mathrm{m}_{0} R}^{n}(M)} x\right)$. Since $H_{\mathrm{m}_{0} R}^{n-1}(M) \in \mathcal{S}$, $\operatorname{Im} f \in \mathcal{S}$ and $\left(0:_{H_{\mathrm{m}_{0} R}^{n}(M)} x\right) \in \mathcal{S}$. Thus $\Gamma_{R_{+}}\left(0:_{H_{\mathrm{m}_{0} R}^{n}}(M)=\right.$ $\left(0:_{\Gamma_{R_{+}}\left(H_{\mathfrak{m}_{0} R}^{n}(M)\right)} x\right) \in \mathcal{S}$. Then $\Gamma_{R_{+}}\left(H_{\mathfrak{m}_{0} R}^{n}(M)\right) \in \mathcal{S}$, because $\Gamma_{R_{+}}\left(H_{\mathfrak{m}_{0} R}^{n}(M)\right)$ is $x$-torsion.

Definition 11. Let $\mathfrak{a}$ be a graded ideal of $R$ and $M \in \mathcal{S}$. Define $t_{\mathfrak{a}}^{\mathcal{S}}(M)=\sup \left\{i \mid H_{\mathfrak{a}}^{i}(M) \notin \mathcal{S}\right\}$ 
Lemma 12. Let $x \in \mathfrak{m}$ be a homogeneous nonzero divisor of $M$. Then

$$
t_{R_{+}}^{\mathcal{S}}\left(\frac{M}{x M}\right) \leq t_{R_{+}}^{\mathcal{S}}(M) .
$$

Proof. Let $\operatorname{deg} x=d$. Since $x$ is a nonzero divisor of $M$, there is an exact sequence

$$
0 \longrightarrow M(-d) \stackrel{x}{\longrightarrow} M \longrightarrow \frac{M}{x M} \longrightarrow 0 .
$$

Application of the functor $H_{R_{+}}^{i}(-)$ to the above exact sequence induces the exact sequence

$$
\begin{aligned}
H_{R_{+}}^{i}(M) & \longrightarrow H_{R_{+}}^{i}\left(\frac{M}{x M}\right) \stackrel{x}{\longrightarrow} H_{R_{+}}^{i+1}(M)(-d) \\
& \longrightarrow H_{R_{+}}^{i+1}(M) .
\end{aligned}
$$

If $t_{R_{+}}^{\mathcal{S}}(M)=n$, then for all $i>n$ we have $H_{R_{+}}^{i}(M) \in \mathcal{S}$. This means that $H_{R_{+}}^{i}(M / x M) \in \mathcal{S}$. Thus, $t_{R_{+}}^{\mathcal{S}}(M / x M) \leq n$.

Remark 13. (1) Any local flat morphism of local Noetherian rings is faithfully flat. So, if $R_{0}$ is flat over $R_{0}$ and $\mathfrak{m}_{0}=\mathfrak{m}_{0} R_{0}$, then $R_{0}$ is faithfully flat over $R_{0}$. Moreover, if $\left(R_{0}, \mathfrak{m}_{0}\right)$ is a faithfully flat local $R_{0}$-algebra, then from ([9], Theorem 1 ) we obtain that $A \in \mathcal{S}$ of the graded $R$-module if and only if $A:=$ $\left(R_{0} \otimes_{R_{0}} A\right) \in \mathcal{S}$ of the graded module over $\hat{R}:=R_{0}^{\prime} \otimes_{R_{0}} R$.

(2) If $\left(\hat{R}_{0}, \mathfrak{m}_{0}\right)$ is a faithfully flat local $R_{0}$-algebra, then $t_{R_{+}}^{\mathcal{S}}(M)=t_{\left(\dot{R}_{0} \otimes_{R_{0}} R\right)_{+}}^{\mathcal{S}}\left(\dot{R}_{0} \otimes_{R_{0}} M\right)$.

Theorem 14. Let $n=t_{R_{+}}^{\mathcal{S}}(M)$; then $H_{R_{+}}^{i}(M) / \mathfrak{m}_{0} H_{R_{+}}^{i}(M) \in \mathcal{S}$ for all $i \geq n$.

Proof. We proceed by induction on $d=\operatorname{dim} R_{0}$. If $i>n$, then $H_{R_{+}}^{i}(M) \in \mathcal{S}$ and then $H_{R_{+}}^{i}(M) / \mathfrak{m}_{0} H_{R_{+}}^{i}(M) \in \mathcal{S}$. Let $i=n$. If $d=1$, then the result follows Theorem 6 . Assume inductively that the result has been proved for all values smaller than $d$, so we prove it for $d$. Let $x$ be an indeterminate and let $R_{0}:=$ $R_{0}[x]_{\mathfrak{m}_{0} R_{0}}, \mathfrak{m}=\mathfrak{m}_{0} R_{0}, R^{\prime}=R_{0} \otimes_{R_{0}} R$ and $M^{\prime}:=R_{0} \otimes_{R_{0}} M$. Then by the flat base change property of local cohomology, we have

$$
\dot{R}_{0} \otimes_{R_{0}} \frac{H_{R_{+}}^{n}(M)}{\mathfrak{m}_{0} H_{R_{+}}^{n}(M)} \cong \frac{H_{\dot{R}_{+}}^{n}(M)}{\mathfrak{m}_{0} H_{\dot{R}_{+}}^{n}(M)} .
$$

Since $R_{0}$ is a faithfully flat local $R_{0}$-algebra, in view of Remark 13(1) it is enough to show that $H_{\dot{R}_{+}}^{n}(M) / \mathfrak{m}_{0} H_{\dot{R}_{+}}^{n}(\dot{M}) \in \mathcal{S}$. Then, we may replace $R$ and $M$ by $R$ and $M$, respectively. Hence we assume that $R_{0} / \mathfrak{m}_{0}$ is infinite residue field. From application of the functor $H_{R_{+}}^{i}(-)$ over the exact sequence

$$
0 \longrightarrow \Gamma_{\mathfrak{m}_{0}}(M) \longrightarrow M \longrightarrow \frac{M}{\Gamma_{\mathfrak{m}_{0}}(M)} \longrightarrow 0,
$$

we have the following exact sequence:

$$
\begin{gathered}
H_{R_{+}}^{i}\left(\Gamma_{\mathfrak{m}_{0}}(M)\right) \stackrel{f}{\longrightarrow} H_{R_{+}}^{i}(M) \stackrel{g}{\longrightarrow} H_{R_{+}}^{i}\left(\frac{M}{\Gamma_{\mathfrak{m}_{0}}(M)}\right) \\
\stackrel{h}{\longrightarrow} H_{R_{+}}^{i+1}\left(\Gamma_{\mathfrak{m}_{0}}(M)\right) .
\end{gathered}
$$

From Lemma 4, we have that $H_{R_{+}}^{i}\left(\Gamma_{\mathfrak{m}_{0}}(M)\right) \in \mathcal{S}$ for all $i$. Now, let $U=\operatorname{Im} f, V=\operatorname{Im} g$, and $W=\operatorname{Im} h$. It follows from Lemma 1 that $\operatorname{Tor}_{i}^{R_{0}}\left(R_{0} / \mathfrak{m}_{0}, U\right)$ and $\operatorname{Tor}_{i}^{R_{0}}\left(R_{0} / \mathfrak{m}_{0}, W\right)$ are in $\mathcal{S}$ for all $i$. If $H_{R_{+}}^{i}\left(M / \Gamma_{\mathfrak{m}_{0}}(M)\right) / \mathfrak{m}_{0} H_{R_{+}}^{\mathrm{i}}\left(M / \Gamma_{\mathfrak{m}_{0}}(M)\right) \in \mathcal{S}$, then $V / \mathfrak{m}_{0} V \in \mathcal{S}$. This implies that $H_{R_{+}}^{i}(M) / \mathfrak{m}_{0} H_{R_{+}}^{i}(M) \epsilon$ $\mathcal{S}$. Since $t^{\mathcal{S}_{R_{+}}(M)}=t^{\mathcal{S}_{R_{+}}}\left(M / \Gamma_{\mathbf{m}_{0}}(M)\right)$, we may assume that $\Gamma_{\mathfrak{m}_{0}}(M)=\Gamma_{\mathfrak{m}_{0} R}(M)=0$. If $x_{0}$ is a nonzero divisor of $M$ and a part of a system of parameters of $\mathfrak{m}_{0}$, then we have the exact sequence

$$
0 \longrightarrow M \stackrel{x_{0}}{\longrightarrow} M \longrightarrow \frac{M}{x_{0} M} \longrightarrow 0 .
$$

Application of the functor $H_{R_{+}}^{n}(-)$ to the above obtained exact sequence induces the exact sequence

$$
\begin{aligned}
& H_{R_{+}}^{n}(M) \stackrel{x_{0}}{\longrightarrow} H_{R_{+}}^{n}(M) \longrightarrow H_{R_{+}}^{n}\left(\frac{M}{x_{0} M}\right) \\
& \stackrel{k}{\longrightarrow} H_{R_{+}}^{n+1}(M) .
\end{aligned}
$$

Let ker $k=X$ and $\operatorname{Im} k=Y$. Since $H_{R_{+}}^{n+1}(M) \in \mathcal{S}$, the graded $R$-module $Y$ is in $\mathcal{S}$ and therefore from Lemma 1 we get $H_{R_{+}}^{n}\left(M / x_{0} M\right) / \mathfrak{m}_{0} H_{R_{+}}^{n}\left(M / x_{0} M\right) \in \mathcal{S}$ if and only if $X / \mathfrak{m}_{0} X \in \mathcal{S}$. On the other hand, application of the functor $R_{0} / \mathfrak{m}_{0} \otimes_{R_{0}}$-induces the exact sequence

$$
\begin{aligned}
\frac{R_{0}}{\mathfrak{m}_{0}} \otimes_{R_{0}} H_{R_{+}}^{n}(M) & \stackrel{p}{\longrightarrow} \frac{R_{0}}{\mathfrak{m}_{0}} \otimes_{R_{0}} H_{R_{+}}^{n}(M) \longrightarrow \frac{X}{\mathfrak{m}_{0} X} \\
& \longrightarrow 0,
\end{aligned}
$$

such that $p:=i d_{R_{0}} / \mathfrak{m}_{0} \otimes_{R_{0}} x_{0}$. Since $x_{0} \in \mathfrak{m}_{0}$, the map $p$ is zero and $H_{R_{+}}^{n}(M) / \mathfrak{m}_{0} H_{R_{+}}^{n}(M) \cong X / \mathfrak{m}_{0} X$. Now, similar to the above arguments, one can conclude that $H_{R_{+}}^{n}(M) / \mathfrak{m}_{0} H_{R_{+}}^{n}(M) \in \mathcal{S}$ if and only if $H_{R_{+}}^{n}\left(M / x_{0} M\right) / \mathfrak{m}_{0} H_{R_{+}}^{n}\left(M / x_{0} M\right) \in \mathcal{S}$. Let $\overline{R_{0}}=R_{0} / x_{0} R_{0}$ and $\overline{\mathfrak{m}_{0}}=\mathfrak{m}_{0} / x_{0} \mathfrak{m}_{0}$. Using the Independence Theorem for graded local cohomology, we have $H_{R_{+}}^{n}\left(M / x_{0} M\right) \cong$ $H_{\left(\overline{R_{0}} \otimes_{R_{0}} R\right)_{+}}^{n}\left(M / x_{0} M\right)$.

Note that the local base ring of the graded ring $\overline{R_{0}} \otimes_{R_{0}} R$ is $\left(\overline{R_{0}}, \overline{\mathfrak{m}_{0}}\right)$ and $\operatorname{dim} \overline{R_{0}}=d-1$. By the following isomorphism

$$
\frac{H_{R_{+}}^{n}\left(M / x_{0} M\right)}{\mathfrak{m}_{0} H_{R_{+}}^{n}\left(M / x_{0} M\right)} \cong \frac{H_{\left(\overline{R_{0}} \otimes_{R_{0}} R\right)_{+}}^{n}\left(M / x_{0} M\right)}{\mathfrak{m}_{0} H_{\left(\overline{R_{0}} \otimes_{R_{0}} R\right)_{+}}^{n}\left(M / x_{0} M\right)}
$$

together with Lemma 12 and using induction hypothesis, this module is in $\mathcal{S}$. 


\section{Conflicts of Interest}

The authors declare that there are no conflicts of interest regarding the publication of this paper.

\section{References}

[1] H. Zöschinger, "Koatomare moduln," Mathematische Zeitschrift, vol. 170, no. 3, pp. 221-232, 1980.

[2] H. Zöschinger, "Minimax-moduln," Journal of Algebra, vol. 102, no. 1, pp. 1-32, 1986.

[3] M. Aghapournahr, "Upper bounds for finiteness of generalized local cohomology modules," J. Alg. Syst, vol. 1, no. 1, pp. 1-9, 2013.

[4] M. Aghapournahr and L. Melkersson, "Local cohomology and Serre subcategories," Journal of Algebra, vol. 320, no. 3, pp. 12751287, 2008.

[5] M. Aghapournahr, A. J. Taherizadeh, and A. Vahidi, "Extension functors of local cohomology modules," Iranian Mathematical Society Bulletin, vol. 37, no. 3, pp. 117-134, 2011.

[6] M. Asgharzadeh and M. Tousi, "Cohen-Macaulayness with respect to Serre classes," Illinois Journal of Mathematics, vol. 53, no. 1, pp. 67-85, 2009.

[7] M. Asgharzadeh and M. Tousi, "A unified approach to local cohomology modules using Serre classes," Canadian Mathematical Bulletin, vol. 53, no. 4, pp. 577-586, 2010.

[8] M. P. Brodmann and R. Y. Sharp, Local Cohomology: An Algebraic Introduction with Geometric Applications, vol. 60 of Cambridge Studies in Advanced Mathematics, Cambridge University Press, Cambridge, England, 1998.

[9] D. Kirby, "Artinian modules and Hilbert polynomials," The Quarterly Journal of Mathematics, vol. 24, pp. 47-57, 1973. 


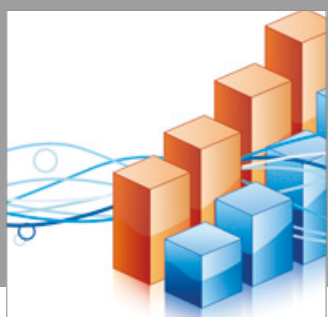

Advances in

Operations Research

vatersals

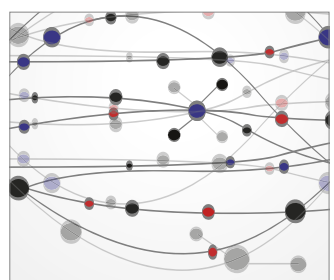

\section{The Scientific} World Journal

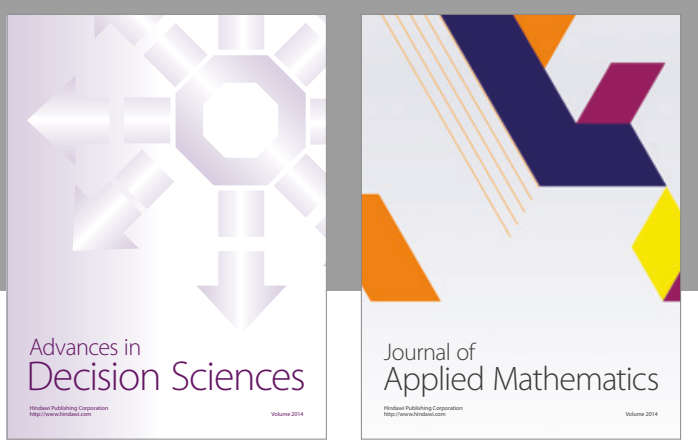

Algebra

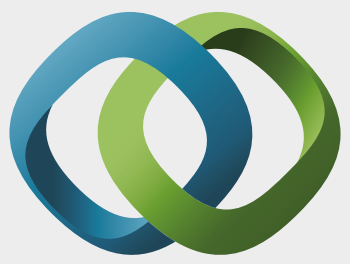

\section{Hindawi}

Submit your manuscripts at

https://www.hindawi.com
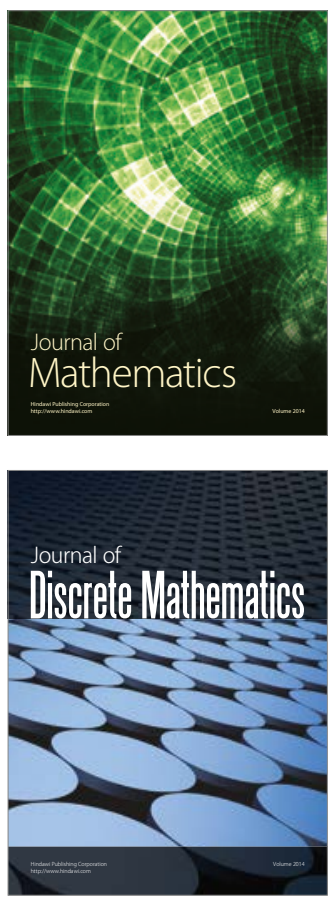

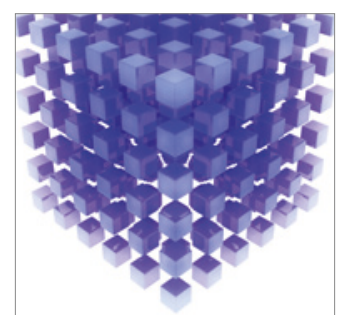

Mathematical Problems in Engineering
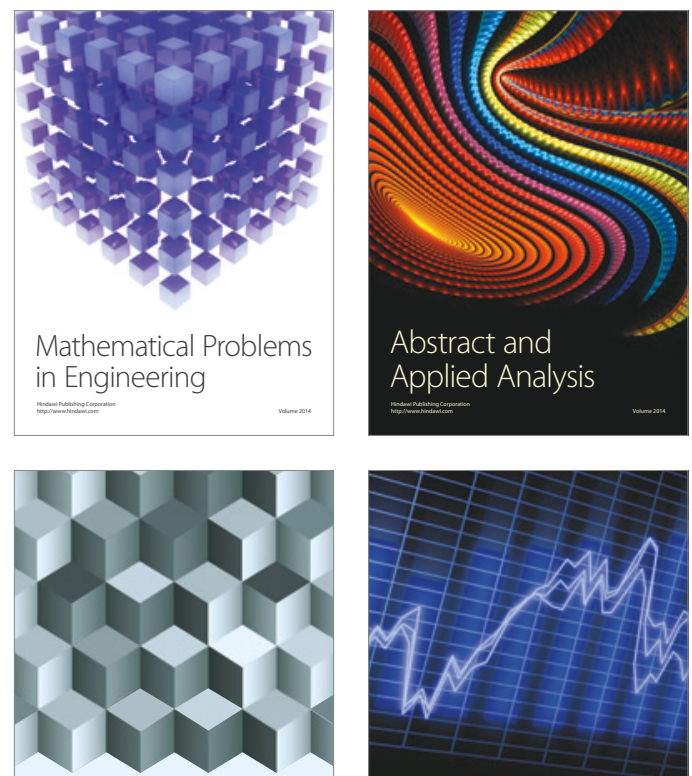

Journal of

Function Spaces



Probability and Statistics
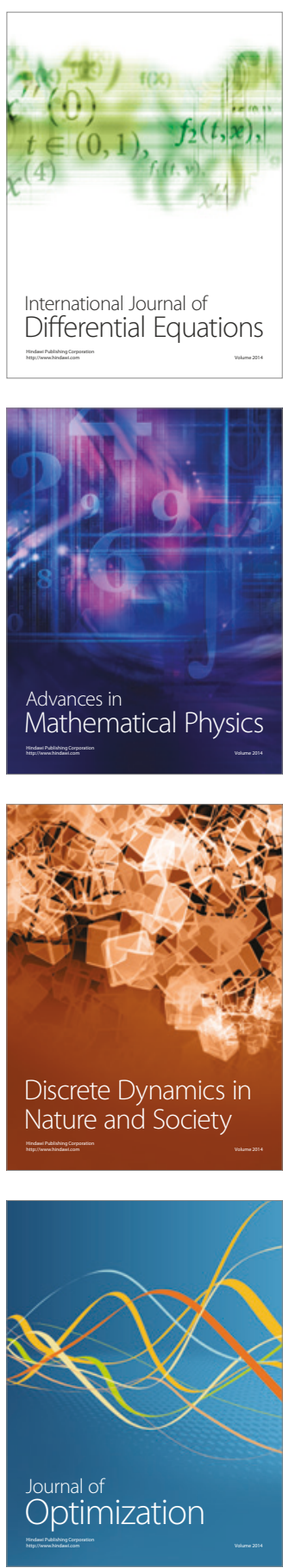